\title{
Resveratrol induces apoptosis in breast cancer cells by E2F1-mediated up-regulation of ASPP1
}

\author{
YUJIE SHI ${ }^{1,2}$, SHIHE YANG $^{2}$, SANDI TROUP $^{2}$, XIN LU $^{3}$, STEVE CALLAGHAN $^{4}$, \\ DAVID S. PARK ${ }^{4}$, YING XING ${ }^{1}$ and XIAOHE YANG $^{2}$
}

\author{
${ }^{1}$ Department of Physiology, College of Basic Medical Science, Zhengzhou University, Zhengzhou \\ 450001, Henan, P.R. China; ${ }^{2}$ Department of Pathology, University of Oklahoma Health Sciences Center, \\ Oklahoma City, OK 73104, USA; ${ }^{3}$ Nuffield Department of Clinical Medicine, Ludwig Institute for Cancer \\ Research, University of Oxford, Oxford OX3 7DQ, UK; ${ }^{4}$ Cellular Molecular Medicine, Ottawa Hospital \\ Research Institute, University of Ottawa, 451 Smyth Road, Ottawa, Ontario K1H 8M5, Canada
}

Received December 8, 2010; Accepted February 1, 2011

DOI: $10.3892 /$ or.2011.1248

\begin{abstract}
Resveratrol is a natural polyphenolic compound with cancer chemopreventive activity. However, our understanding of the molecular mechanism responsible for resveratrolinduced apoptosis is still very limited. Here, we used MCF-7 and MDA-MB231 breast cancer cells as a model to demonstrate that resveratrol induced the expression of ASPP1, a new member of the ASPP (apoptosis stimulation protein of p53) family, which plays an important role in the regulation of apoptosis. Moreover, resveratrol enhanced apoptosis of MCF-7/ASPP1 cells, accompanied by higher expression of Bax and p21. In contrast, siRNA-mediated knockdown of ASPP1 inhibited apoptosis in MB231 cells. Furthermore, we found that higher levels of ASPP1 were associated with adenovirus-mediated overexpression of E2F1 while siRNAmediated E2F1 knockdown led to down-regulation of ASPP1. In conclusion, our results demonstrate that overexpression of ASPP1 rendered MCF-7 and MDA-MB231 breast cancer cells more sensitive to resveratrol-mediated apoptosis via the E2F pathway, thus suggesting that ASPP1 may represent a novel therapeutic target for resveratrol in human breast cancer.
\end{abstract}

\section{Introduction}

Resveratrol (3,5,4'-trihydroxystilbene) is a natural polyphenolic compound that is commonly found in grape skin,

Correspondence to: Dr Ying Xing, Department of Physiology, College of Basic Medical Science, Zhengzhou University, Zhengzhou 450001, Henan, P.R. China

E-mail: xingy@zzu.edu.cn

Dr Xiaohe Yang, Present address: Julius L. Chambers Biomedical Biotechnology Research Institute, North Carolina Central University, Kannapolis, NC 28081, USA

E-mail: xyang@nccu.edu

Key words: resveratrol, apoptosis stimulation protein of $\mathrm{p} 53$, apoptosis, E2F-1, MDA-MB231, MCF-7, breast cancer red wine, peanuts and some Chinese herbs (1-4). Previous studies have demonstrated that resveratrol has a number of biological activities that might be beneficial to human health, including anti-oxidant (5-7), anti-inflammatory $(8,9)$, cardioprotective (10-14) and phytoestrogen properties (15-18). Importantly, resveratrol may also act as an anti-cancer agent by inhibiting cell proliferation/transformation and inducing apoptosis. Although resveratrol mediated growth inhibition and apoptosis induction has been associated with a number of molecules and pathways, the precise mechanisms of resveratrol mediated activities remain unclear. This is exemplified by the regulation of p53 pathway by resveratrol.

p53 is a tumor suppressor mutated in about $50 \%$ of human cancers. Activation of p53 pathway plays a critical role in homeostatic maintenance and cellular responses to anti-cancer therapeutics. Regulation of the p53 pathway in resveratrol induced apoptosis appears to be complicated. It was demonstrated that resveratrol induced apoptosis in cells expressing wild-type p53 (p53+/+), but not in p53-deficient (p53-/-) cells. Several studies, however, indicated that some cancer cells with mutant p53 remain sensitive to resveratrol (19). Since the p53 pathway can also be regulated by other p53 family members and p53 interacting proteins, such as p73, p63 and the ASPP family members, it is important to understand how these p53 interacting molecules are regulated in resveratrol induced apoptosis.

ASPP1 is a member of the ASPP (apoptosis stimulation protein of p53) family, which also includes ASPP2 and iASPP. This family is characterized by sharing a highly conserved carboxyl terminus, which contains a proline-rich region, four ankyrin repeats and an SH3 domain, and p53 binding capacity. Mechanistic study revealed that ASPP1 and ASPP2 proteins enhance the apoptotic function of p53 by stimulating the selection of proapoptotic promoters, whereas iASPP-p53 interaction inactivates p53 mediated apoptosis. As putative tumor suppressors, ASPP1 and ASPP2 are frequently down-regulated in human breast tumors and leukemia (20), which may involve the hypermethylation of its promoter. Importantly, ASPP1 and ASPP2 may also interact with p73 or 
p63 and stimulate p73 or p63 mediated apoptosis in a manner similar to p53, which underscores the role of ASPP1 and ASPP2 in tumor cells with different p53 status. Nevertheless, the role of ASPP proteins in resveratrol induced apoptosis has not been documented.

In this study, we addressed the function and regulation of ASPP1 in resveratrol treated breast cancer cells. Our results indicate that resveratrol up-regulates the expression of ASPP1 through E2F-1 mediated transcription and resveratrol induced ASPP1 expression plays a critical role in resveratrol induced apoptosis.

\section{Materials and methods}

Cell lines and cell culture. MCF-7 and MDA-MB-231 cell lines were from American Type Culture Collection (ATCC). MCF-7/C and MCF-7/ASPP1 cells were stable MCF-7 cell lines transfected with pCDNA3 vector or ASPP1-V5/pcDNA3 encoding plasmid after G418 selection. The cells were cultured in DMEM/F12 medium containing $10 \%$ fetal bovine serum (FBS). To prepare for protein lysate, the cells were seeded into $60-\mathrm{mm}$ dish at $1 \times 10^{6} / \mathrm{dish} 24 \mathrm{~h}$ before treatment with resveratrol or $0.1 \%$ DMSO (solvent for resveratrol) as control at the indicated time.

MTT assay. The cells were seeded into 24-well-plate at $3 \times 10^{3}$ cells/well $24 \mathrm{~h}$ before treatment with resveratrol at indicated concentrations for 6 days. After removal of the medium, $50 \mu 1$ of MTT solution $(500 \mu \mathrm{g} / \mathrm{ml})$ was added to each well followed by $4-\mathrm{h}$ incubation. MTT solution was replaced by DMSO to dissolve blue formazan crystals, and absorbance was measured at $562 \mathrm{~nm}$ using a microplate reader. Each group contains 4 parallel samples. Survival fractions were calculated as the absorbance in the treated cells over the control (treated with $0.1 \%$ DMSO). Statistical differences were analyzed with Student's t-test.

siRNA transfection. Specific or control siRNAs (Santa Cruz Biotechnology Inc., Santa Cruz, CA) were transfected into the cells using Lipofectamine 2000 (Invitrogen, Carlsbad, CA) according to the manufacturer's protocol.

Adenovirus infection. After $18 \mathrm{~h}$ of plating, MCF-7 or MB-231 cells were infected with adenovirus $(\mathrm{MOI}=150)$. The cells were treated with $50 \mu \mathrm{M}$ resveratrol $2 \mathrm{~h}$ after infection.

RNA extraction and reverse transcription-PCR (RT-PCR). Total RNA was isolated from the cells using Rneasy Mini Kit (Qiagen, Carlsbad, CA). First strand cDNA synthesis was performed using SuperscriptIII ${ }^{\mathrm{TM}}$ First Strand synthesis system (Invitrogen) with $3 \mu \mathrm{g}$ of total RNA, one tenth of the RT product was used as template for PCR reaction using the following primers: ASPP1 forward, 5'-gca gca cac agc gcc tta aat aag-3', reverse, 5'-tcc att gtc cac atc ggc caa ggt-3' annealing at $60^{\circ} \mathrm{C}$; bax forward, $5^{\prime}$-tgc ttc agg gtt tca tcc ag- $3^{\prime}$ reverse, $5^{\prime}$-ggc ggc aat cat cct ctg- $3^{\prime}$ annealing at $60^{\circ} \mathrm{C}$; p21 forward, 5'-ctg gag act ctc agg gtc gaa-3', reverse, 5'-ggc gtt tgg agt ggt aga aat ct- $3^{\prime}$ annealing at $55^{\circ} \mathrm{C}$. After PCR for 30 cycles, $20 \mu \mathrm{l}$ of PCR products were electrophoresed on $2 \%$ agarose gel and visualized by Ethidium Bromide staining.
The image was acquired and analyzed using UPVII gel documentation system.

Western blot analysis. The cells with different treatments were collected and lysed with lysis buffer on ice for $30 \mathrm{~min}$. Cell lysate was prepared by centrifugation and the protein concentration was determined using BCA assay kit (Pierce Biotechnology Inc, IL). Protein lysate $(50 \mu \mathrm{g})$ was separated with 10 or $12 \%$ SDS-PAGE and transferred to nitrocellulose membrane. The membrane was probed with a specific primary antibody, followed by washing and probing with a corresponding secondary antibody. The specific protein band was visualized by autoradiography using an ECL kit (Pierce Biotechnology).

Apoptosis detection by ELISA. Induction of apoptosis was assessed with a cell death detection ELISA kit (Roche Diagnostics) following the manufacturer's instruction. In brief, cells in a 24-well plate were treated with resveratrol at indicated concentrations for $30 \mathrm{~h}$. Both floating and adherent cells were collected for lysis preparation and detected at the absorbance at $405 \mathrm{nM}$.

Statistical analysis. The data are expressed as the mean \pm S.E.M. Student's t-test was used to determine the level of significance and a P-value $<0.05$ was regarded as significant.

\section{Results}

Resveratrol induces apoptosis of breast cancer cells. As the first step to examine the possible anticancer effects of resveratrol on human breast cancer cells, we employed MTT assay and found that treatment of MCF-7 or MDA-MB-231 breast cancer cells with resveratrol led to significant decrease of cell viability in a dose-dependent manner (Fig. 1A). Next, we aimed to address whether decreased cell viability is due to increased apoptosis upon resveratrol treatment since proapoptotic function of resveratrol has been documented. To this end, we performed Western blot analysis to examine the level of cleaved PARP, a hallmark of apoptosis, in resveratrol treated breast cancer cells. The results demonstrate that resveratrol incuded the production of cleaved PARP in MCF-7 and MB231 cells in a dose dependent manner and the effect was more significant in MB231 cells (Fig. 1B). Taken together, these results indicate that resveratrol inhibits the cell viability of MCF-7 and MDA-MB-231 breast cancer cells by inducing apoptosis.

Resveratrol induces the expression of ASPP1 in breast cancer cells. To gain more insights into the molecular mechnisms by which resveratrol induces apoptosis in breast cancer cells, we examined the expression of ASPP1 since ASPP1 enhances the apoptotic function of p53. Western blot analysis demonstrated that resveratrol treatment led to significantly increased protein level of ASPP1 in MCF-7 cells in a dosedependent manner, and to a lesser extent in MB231 cells as well (Fig. 2A). To confirm the up-regulation of ASPP1 by resveratrol, we employed RT-PCR and luciferase assay to dertermine the expression of ASPP1 at mRNA level and 
A

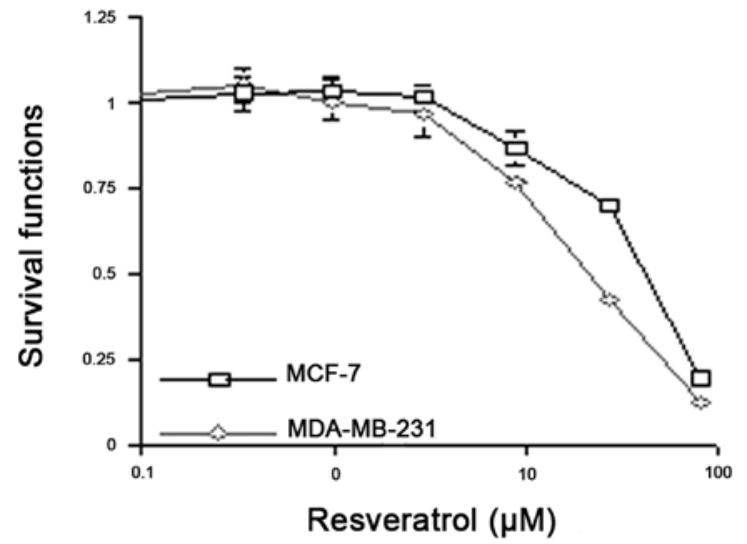

B MCF-7

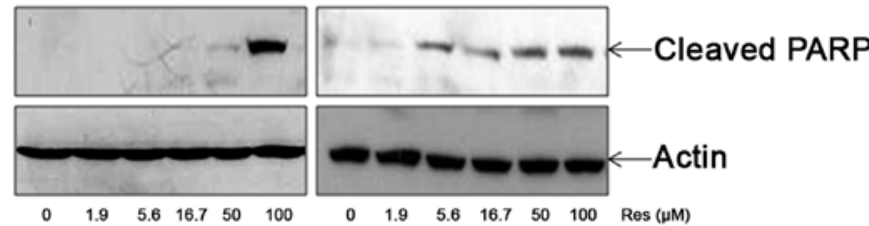

Figure 1. Resveratrol inhibits cell viability of breast cancer cells by inducing apoptosis. (A) Resveratrol decreases cell viability in MCF-7 and MDA-MB231 human breast cancer cells as determined by MTT assay. (B) Resveratrol promotes apoptosis in MCF-7 and MDA-MB-231 human breast cancer cells as determined by Western blot analysis of cleaved PARP.

found that resveratrol induced increased mRNA level of ASPP1 in MCF-7 cells in a dose-dependent manner, and to a lesser extent in MB231 cells as well (Fig. 2B). Collectively, these data demonstrate that resveratrol induces the expression of ASPP1 in breast cancer cells.

ASPP1 sensitizes breast cancer cells to resveratrol-induced apoptosis. Having established that resveratrol induces apoptosis in breast cancer cells on one hand and induces the expression of ASPP1 in breast cancer cells on the other hand, we further addressed the functional role of up-regulation of ASPP1 in resveratrol-induced apoptosis. We employed MCF-7 cells as the model system since the up-regulation of ASPP1 is significantly higher in MCF-7 cells than in MB231 cells (Fig. 2). First we established a stable MCF-7 cell line that overexpresses ASPP1 and named it MCF-7/ASPP1. We also made stable MCF-7 cell line transfected with pCDNA3 vector and named it MCF-7/C to serve as control (Fig. 3A). Next, we treated MCF-7/ASPP1 or MCF-7/C cells with resveratrol and examined the cell survival and apoptosis rate in these cells by MTT assay and apoptosis ELISA assay, respectively. The results showed that upon treatment with increasing concentrations of resveratrol MCF-7/ASPP1 cells exhibited much lower survival rate and much higher apoptosis rate than MCF-7/C cells (Fig. 3B and C). Next, we performed RT-PCR asalysis to address whether increased level of ASPP1 could modulate p53 target genes such as Bax and p21 to promote resveratrolinduced apoptosis, and indeed found higher mRNA levels of Bax and p21 in MCF-7/ASPP1 cells than in MCF-7/C cells upon resveratrol treatment (Fig. 3D). Taken together, these results indicate that ASPP1 sensitizes breast cancer cells to
A

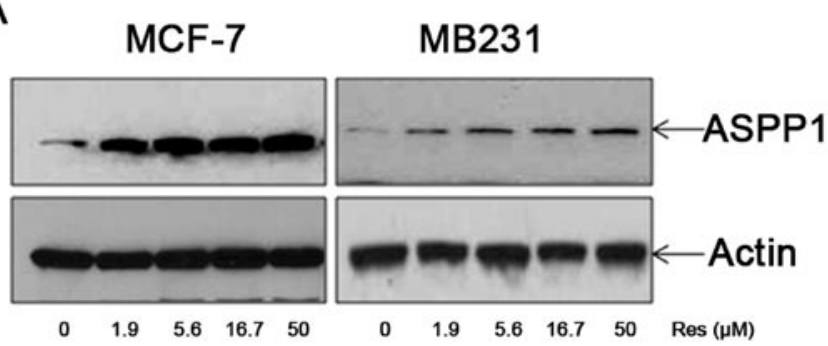

B

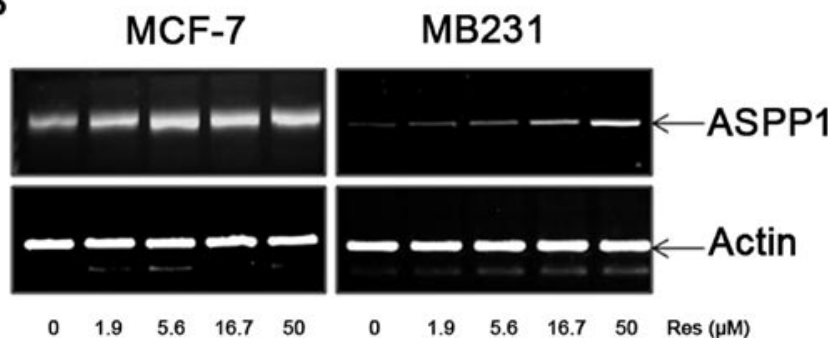

Figure 2. Resveratrol induces the expression of ASPP1 in breast cancer cells MCF-7 and MB231 were treated with resveratrol at the indicated doses for $48 \mathrm{~h}$. The treated cells were then analyzed for protein level of ASPP1 by Western blot analysis (A) or mRNA level of ASPP1 by RT-PCR assay (B). Actin served as internal control.

resveratrol-induced apoptosis, at least in part by up-regulating p53 target genes Bax and p21.

ASPP1 synergizes with caspase-3 in resveratrol-induced apoptosis in breast cancer cells. As the next step to confirm the function of ASPP1 in resveratrol-induced apoptosis, we employed a stable MCF-7 cell line that overexpresses caspase-3, i.e. MCF-7/caspase-3 that we made previously (21) and assessed the effect of ASPP1 expression on resveratrolinduced apoptosis of MCF-7/caspase-3 cells. As shown in Fig. 4A and B, compared with MCF-7 cells, resveratrol induced significantly higher apoptosis rate and lower survival rate of MCF-7/caspase-3 cells, and this was dose-dependent. These results suggest that resveratrol induces apoptosis of breast cancer cells via classic apoptotic pathway. Furthermore, when ASPP1 was overexpressed in MCF-7/caspase-3 cells, ASPP1 synergized with caspase-3 to induce apoptosis, leading to significantly reduced cell survival compared with untransfected MCF-7/caspase-3 cells. The synergism between ASPP1 and caspase-3 to induce apoptosis and inhibit cell survival was more evident when MCF-7/caspase-3 cells were treated with resveratrol (Fig. 4C). Collectively, these findings demonstrate that ASPP1 synergizes with caspase-3 in resveratrol-induced apoptosis in breast cancer cells.

Resveratrol induces the expression of ASPP1 via E2F-1 pathways. As the final step to characterize the molecular mechnisms by which resveratrol induces the expression ASPP1, we focused on E2F1 since previous studies indicate ASPP1 as a target of E2F1 $(22,23)$. First, we inspected the expression of E2F1 at both mRNA and protein levels in breast cancer cells upon resveratrol treatment. The results showed that resveratrol induced the expression of E2F1 in 
A

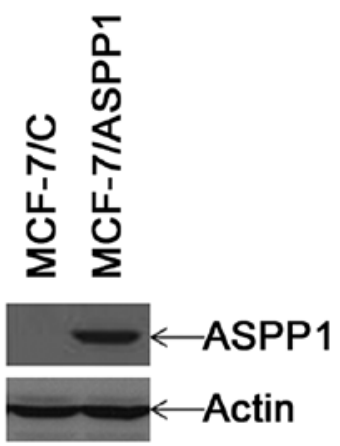

B

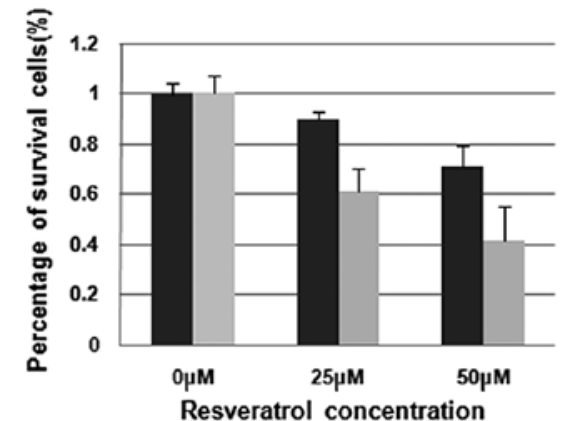

C
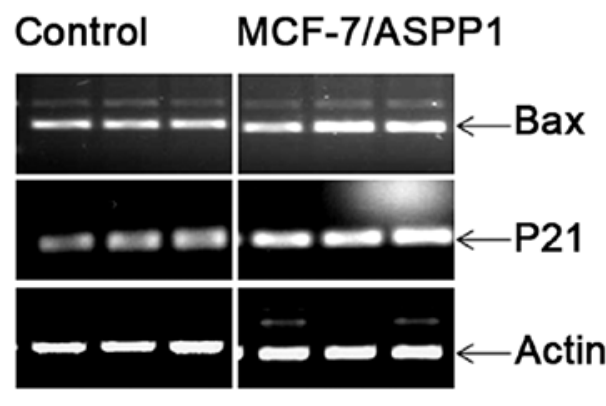

D

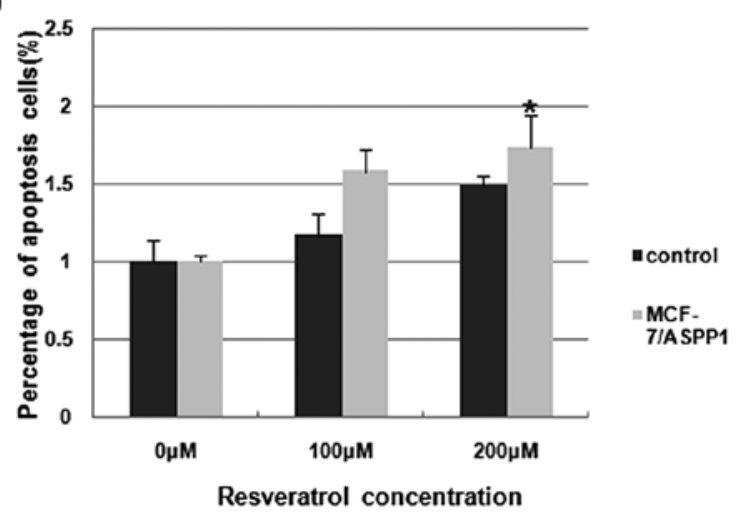

Figure 3. ASPP1 sensitizes MCF-7 cells to resveratrol-induced apoptosis. (A) Western blot analysis of ASPP1 level in MCF-7/ASPP1 cells and control MCF-7/C cells. Actin served as internal control. (B) MCF-7/C and MCF-7/ ASPP1 cells were treated with resveratrol at the indicated concentrations for 5 days and then subjected to MTT assay. Survival fractions of the treated cells, based on 6 parallel samples, were analyzed. (C) MCF-7/C and MCF-7/ ASPP1 cells were treated with resveratrol at the indicated concentrations for 5 days and then subjected to Apoptosis ELISA assay. The percentage of apoptosis is shown as the mean \pm SD of quadruplicate samples. (D) MCF-7/C and MCF-7/ASPP1 cells were treated with resveratrol at the indicated doses for $48 \mathrm{~h}$. The treated cells were then analyzed for mRNA levels of Bax and p21 by RT-PCR assay. Actin served as internal control.

both MCF-7 and MB231 cells in a dose-dependent manner (Fig. 5A and B). Next we aimed to explore the functional role of E2F1 in the induction of ASPP1 in resveratrol-treated

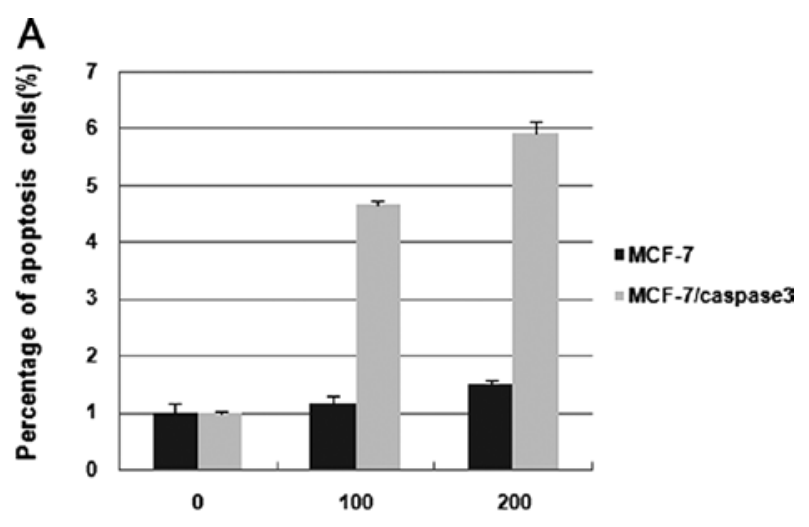

Resveratrol concentration $(\mu \mathrm{M})$

B

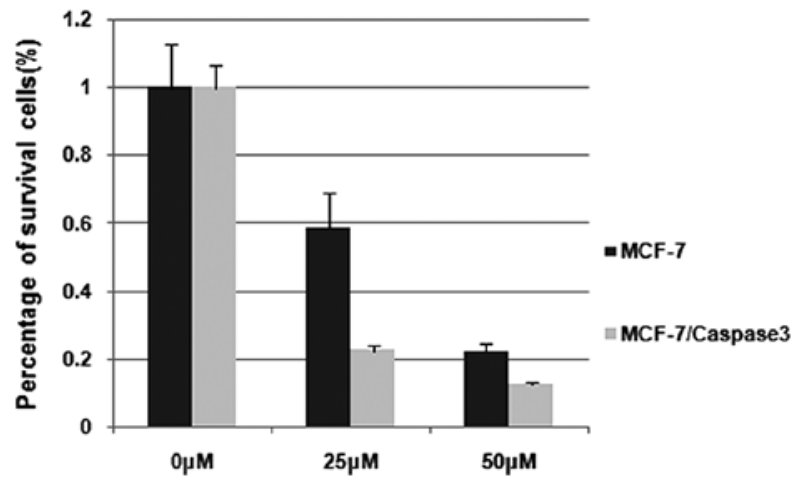

Resveratrol concentration

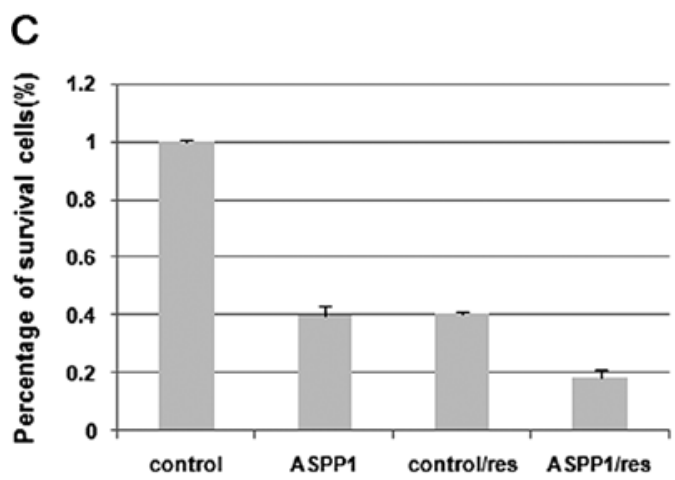

MCF-7/Caspase3 transient transfect ASPP1

Figure 4. ASPP1 synergizes with caspase-3 in resveratrol-induced apoptosis in breast cancer cells. (A) MCF-7/C and MCF-7/caspase- 3 cells were treated with resveratrol at the indicated concentrations for 5 days and then subjected to Apoptosis ELISA assay. The percentage of apoptosis is shown as the mean \pm SD of quadruplicate samples. (B) MCF-7/C and MCF-7/caspase-3 cells were treated with resveratrol at the indicated concentrations for 5 days and then subjected to MTT assay. Survival fractions of the treated cells, based on 6 parallel samples, were statistically analyzed. "P<0.01. (C) MCF-7/C and MCF-7/caspase-3 cells were transiently transfected with ASPP1 expression plasmid and then either left untreated or treated with resveratrol for 5 days and subjected to MTT assay. Survival fractions of the treated cells, based on 6 parallel samples, were statistically analyzed. ${ }^{*} \mathrm{P}<0.01$.

breast cancer cells. For this purpose, we employed both gain and loss of approaches to overexpress or knock down E2F1 in the cells and examine the effect on the expression level of ASPP1. Our resutls demonstrate that siRNA-mediated 
A

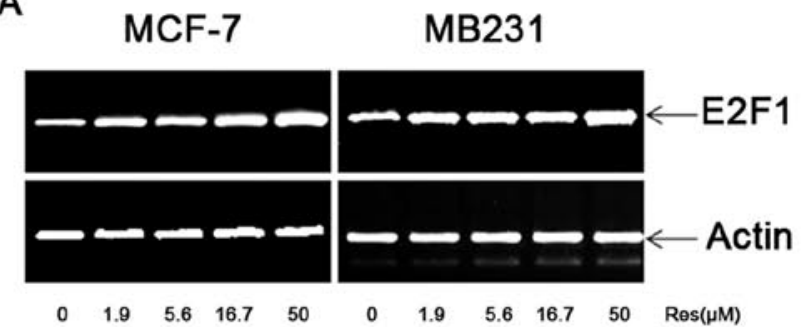

B

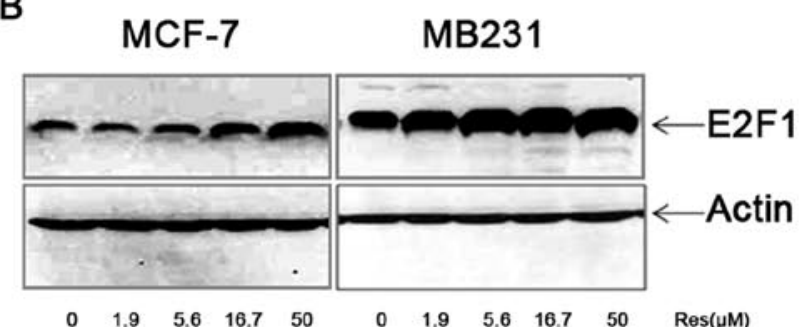

C

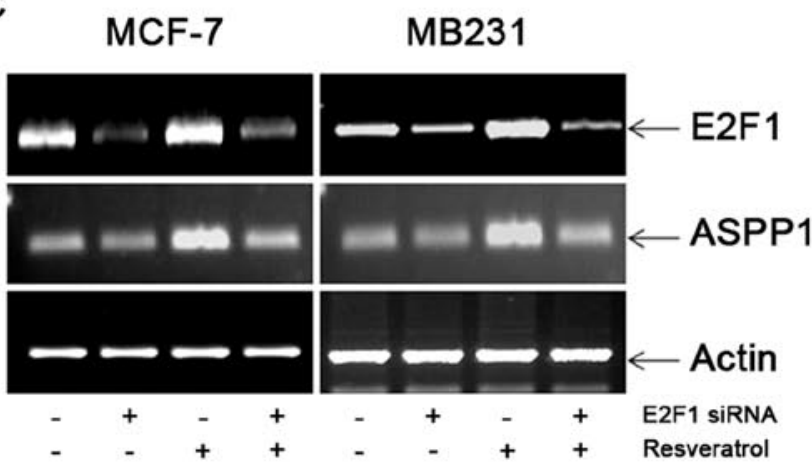

D

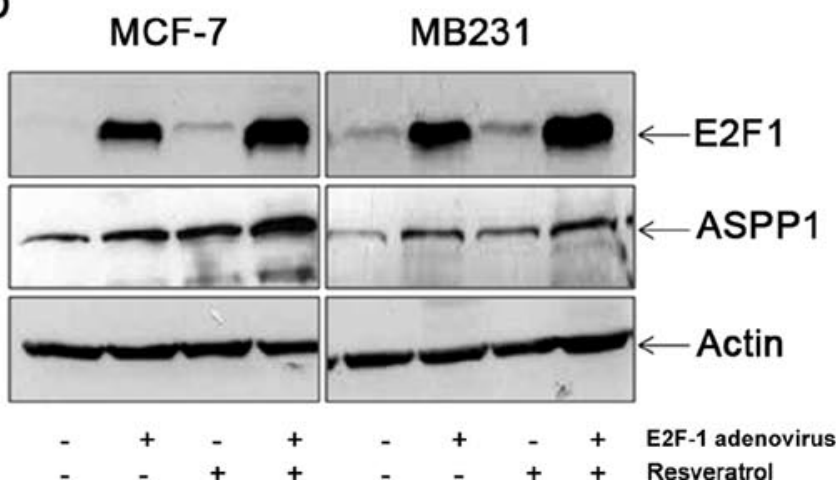

Figure 5. Resveratrol induces the expression of ASPP1 via E2F-1. (A and B) MCF-7 and MB231 were treated with resveratrol at the indicated doses for $48 \mathrm{~h}$. The treated cells were then analyzed for mRNA level of E2F-1 by RT-PCR assay (A) or protein level of E2F-1 by Western blot analysis (B). Actin served as internal control. (C) MCF-7 and MB231 were transfected with E2F-1 siRNA or control siRNA for $24 \mathrm{~h}$ and then either untreated or treated with $50 \mu \mathrm{M}$ resveratrol for $24 \mathrm{~h}$. The cells were then analyzed for mRNA levels of E2F-1 and ASPP1 by RT-PCR assay. Actin served as internal control. (D) MCF-7 and MB231 were transduced with E2F-1 adenovirus or control adenovirus $(2 \mathrm{~h})$ and then either left untreated or treated with $50 \mu \mathrm{M}$ resveratrol for $24 \mathrm{~h}$. The cells were analyzed for protein levels of E2F-1 and ASPP1 by Western blot analysis. Actin served as internal control.

knockdown of E2F1 led to reduced mRNA level of ASPP1 in both MCF-7 and MB231 cells in the presence of resveratrol (Fig. 5C). In contrast, adenovirus-mediated overexpression of E2F-1 led to increased protein level of ASPP1 in these two cell lines (Fig. 5D). Thus, these data suggest that resveratrol induces the expression of ASPP1 in breast cancer cells via E2F-1.

\section{Discussion}

Resveratrol has been shown to be an efficient anticancer nutrient that inhibits cancer initiation and promotion (2). Overwhelming evidence has demonstrated that resveratrol modulates multiple signaling pathways regulating the cell cycle and inducing apoptosis (24). Several studies using different cancer cell models suggest that resveratrol induces apoptosis of cancer cells in a p53 dependent manner (25-28). Nevertherless, it is well known that more than $50 \%$ of human tumors contain a mutation or deletion of p53 gene (29). This raises an important question whether resveratrol induces apoptosis of cancer cells in a p53-independent manner and bear significance on the clinical application of resveratrol for the treatment of cancers with p53 mutations.

Therefore, in this study we employed two different breast cancer cell lines, MCF-7 that harbors wild-type p53 and MDA-MB-231 that harbors mutant p53 to address whether resveratrol affects the survival and apoptosis of cancer cells regardless of their p53 status. MTT assay showed that reveratrol had inhibitory effect on the growth of both MCF-7 and MB231 cells, leading to reduction in the cell survival rate in these two cell lines in a dose-dependent manner. Furthermore, Western blot analysis of cleaved PARP demonstrated that resveratrol induced the cleavage of PARP in both MCF-7 and MB231 cells in a dose-dependent manner. These results suggest that resveratrol promotes the apoptosis of both MCF-7 and MB231 cells, thus contributing to the decreased cell survival rate as revealed by MTT assay. Taken together, these results suggest that resveratrol induced apoptosis of breast cancer cells independent of their p53 status. Even when p53 is mutated as it occurs in MB231 cells, resveratrol still exhibits anti-tumor effect by inducing apoptosis.

Increasing evidence suggests that ASPP family member ASPP1 and ASPP2 not only play an important role in p53-mediated apoptosis (30), but also induce apoptosis via p53 homologues p63 and p73 (31,32). Interestingly, the expression of ASPP1 and ASPP2 is frequently down-regulated in human breast tumors. By semi-quantitative RT-PCR analysis of the mRNA levels of ASPP1 and ASPP2 in a panel of paired normal and tumor samples derived from 58 breast cancer patients among which 40 breast carcinomas express wild-type p53 while the other 18 express mutant p53, it was found that among the 40 carcinoma samples with wild-type p53, reduced expression of ASPP1 and ASPP2 were detected in 24 and 9 samples, respectively, and 8 of 9 tumors with reduced expression of ASPP2 also showed reduced expression of ASPP1. On the other hand, among the 18 tumor samples with mutant p53, 3 and 2 tumors showed reduced expression of ASPP1 and ASPP2, respectively. These data indicate that there is a selective advantage for tumor cells to lose the expression of ASPP1 and ASPP2 and support the notion that the ASPP family functions as an important tumor suppressor in breast cancer development (33). 
Consistent with previous studies, in this study we found that the expression of ASPP1 at the mRNA and protein levels was low in MCF-7 and MB231 breast cancer cells. Most notably, we observed for the first time that resveratrol induced the up-regulation of ASPP1 at the mRNA and protein levels in a dose-dependent manner and this was positively correlated with resveratrol-induced apoptosis in both MCF-7 and MB231 cells. To investigate the functional role of up-regulation of ASPP1 in resveratrol-induced apoptosis, we employed both gain of function and loss of function approaches to demonstrate that overexpression of ASPP1 in MCF-7 cells promoted resveratrol-induced apoptosis while knockdown of ASPP1 in MB231 cells inhibited resveratrol-induced apoptosis. Furthermore, we employed a stable MCF-7 cell line that overexpress caspase-3, a crucial mediator of classic apoptosis pathway, to demonstrate that ASPP1 synergizes with caspase-3 in resveratrol-induced apoptosis. Taken together, our results provide strong support for the essential role of ASPP1 in resveratrol-induced apoptosis of breast cancer cells.

To provide insight into the molecular mechanims by which resveratrol-induced ASPP1 up-regulation modulates apoptosis, we examined the expression of p53 target genes such as Bax and p21 that are involved in apoptosis and observed higher mRNA levels of Bax and p21 in ASPP1 overexpressing MCF-7 cells compared with normal MCF-7 cells. These results suggest that ASPP1 might contribute to the up-regulation of p53 target genes Bax and p21, thereby sensitizing breast cancer cells to resveratrol-induced apoptosis.

Finally, to elucidate the molecular mechnisms whereby resveratrol induces the up-regulation of ASPP1, we first established that resveratrol induced the expression of E2F-1 in both MCF-7 and MB231 cells in a dose-dependent manner given that ASPP1 is a target gene of E2F-1. Next via both gain and loss approaches we found that adenovirus-mediated overexpression of E2F-1 led to increased protein level of ASPP1 while siRNA-mediated knockdown of E2F-1 led to reduced mRNA level of ASPP1 in both MCF-7 and MB231 cells in the presence of resveratrol. These results reveal that E2F-1 pathway downstream of resveratrol treatment contributes to the up-regulation of ASPP1 in breast cancer cells.

In conclusion, in this study we present a novel scenario how resveratrol sensitizes breast cancer cells to apoptosis, i.e. resveratrol induces the expression of transcription factor E2F-1, E2F-1 then drives the expression of ASPP1, and ASPP1 promotes apoptosis of breast cancer cells via two independent pathways. In cells with wild-type p53 such as MCF-7 cells, ASPP1 enhances the expression of p53 target genes Bax and p21 to induce apoptosis. In cells with mutant p53 such as MB231 cells, ASPP1 may cooperate with p53 homologues p63 or p73 to activate downstream target genes to induce apoptosis. Whatever the machanims, our new findings that resveratrol induces the up-regulation of ASPP1 in breast cancer cells suggest that ASPP1 may represent a novel therapeutic target for resveratrol in breast cancer.

\section{Acknowledgements}

This study was supported in part by a Research Scholar grant from the American Cancer Society (ACS) and a Health Research grant from the Oklahoma Center for the Advancement of Science and Technology (OCAST) to X.Y.

\section{References}

1. Takaoka MJ: Of the phenolic substances of white hellebore (Veratrum grandiflorum Loes. fil). Hokkaido Imperial University. J Faculty Sci 3: 1-16, 1940.

2. Jang M, Cai L, Udeani GO, et al: Cancer chemopreventive activity of resveratrol, a natural product derived from grapes. Science 275: 218-220,1997.

3. Burns J, Yokota T, Ashihara H, et al: Plant foods and herbal sources of resveratrol. J Agric Food Chem 50: 3337-3340, 2002.

4. Le Corre L, Chalabi N, Delort L, et al: Resveratrol and breast cancer chemoprevention: molecular mechanisms. Mol Nutr Food Res 49: 462-471, 2005.

5. Fremont, L, Belguendouz L and Delpal S: Antioxidant activity of resveratrol and alcohol-free wine polyphenols related to LDL oxidation and polyunsaturated fatty acids. Life Sci 64: 2511-2521, 1999

6. Morales AI: Protective effect of trans-resveratrol on gentamicininduced nephrotoxicity. Antioxid Redox Signal 4: 893-898, 2002.

7. Baxter RA: Anti-aging properties of resveratrol: review and report of a potent new antioxidant skin care formulation. J Cosmet Dermatol 7: 2-7, 2008.

8. Chen G, Shan W, Wu Y, Ren L, Dong J and Ji Z: Synthesis and anti-inflammatory activity of resveratrol analogs. Chem Pharm Bull (Tokyo) 53: 1587-1590, 2005.

9. Udenigwe CC, Ramprasath VR, Aluko RE, et al: Potential of resveratrol in anticancer and anti-inflammatory therapy. Nutr Rev 66: 445-454, 2008.

10. Renaud S and de Lorgeril M: Wine, alcohol, platelets, and the French paradox for coronary heart disease. Lancet 339: 1523-1526, 1992.

11. Ertelli AA: Antiplatelet activity of synthetic and natural resveratrol in red wine. Int J Tissue React 17: 1-3,1995.

12. Wang Z: Effects of red wine and wine polyphenol resveratrol on platelet aggregation in vivo and in vitro. Int J Mol Med 9: 77-79, 2002.

13. Bohm M, Rosenkranz S and Laufs U: Alcohol and red wine: impact on cardiovascular risk. Nephrol Dial Transplant 19: 11-16, 2004.

14. Marques FZ, Markus MA and Morris BJ: Resveratrol: cellular actions of a potent natural chemical that confers a diversity of health benefits. Int J Biochem Cell Biol 41: 2125 2128, 2009.

15. Gehm BD, McAndrews JM, Chien PY and Jameson JL: Resveratrol, a polyphenolic compound found in grapes and wine, is an agonist for the estrogen receptor. Proc Natl Acad Sci USA 94: 14138-14143, 1997.

16. Lu R and Serrero G: Resveratrol, a natural product derived from grape, exhibits antiestrogenic activity and inhibits the growth of human breast cancer cells. J Cell Physiol 179: 297-304, 1999.

17. Bowers JL, Tyulmenkov VV, Jernigan SC and Klinge CM: Resveratrol acts as a mixed agonist/antagonist for estrogen receptors alpha and beta. Endocrinology 141: 3657-3667, 2000.

18. Bhat KP and Pezzuto JM: Resveratrol exhibits cytostatic and anti-estrogenic properties with human endometrial adenocarcinoma (Ishikawa) cells. Cancer Res 61: 6137-6144, 2001.

19. Ling X, He X, Apontes $P$, et al: Enhancing effectiveness of the MDR-sensitive compound T138067 using advanced treatment with negative modulators of the drug-resistant protein survivin. Am J Transl Res 1: 393-405, 2009.

20. Samuels-Lev Y, O'Connor DJ, Bergamaschi D, et al: ASPP proteins specifically stimulate the apoptotic function of $\mathrm{p} 53$ Mol Cell 8: 781-794, 2001.

21. Yang XH, Liu X, Sladek TL, et al: Reconstitution of caspase-3 sensitizes MCF-7 breast cancer cells to doxorubicin- and etoposide-induced apoptosis. Cancer Res 61: 348-354, 2001.

22. Stanelle J, Stiewe T, Theseling CC, et al: Gene expression changes in response to E2F-1 activation. Nucleic Acids Res 30: 1859-1867, 2002.

23. Chen D, Padiernos E, Ding F, et al: Apoptosis stimulating protein of p53-2 (ASPP2/53BP2L) is an E2F target gene. Cell Death Differ 12: 358-368, 2005. 
24. Signorelli $\mathrm{P}$ and Ghidoni R: Resveratrol as an anticancer nutrient: molecular basis, open questions and promises. J Nutr Biochem 16: 449-466, 2005.

25. She Q, Bode A, Ma WY, et al: Resveratrol-induced activation of p53 and apoptosis is mediated by extracellular-signal-regulated protein kinases and p38 kinase. Cancer Res 61: 1604-1610, 2001.

26. Kuo P, Chiang L and Lin CC: Resveratol-induced apoptosis is mediated by p53-dependent pathway in HepG2 cells. Life Sci 72: 23-34, 2002

27. Laux MT, Aregullin M, Berry JP, et al: Identification of a p53-dependent pathway in the induction of apoptosis of human breast cancer cells by the natural product, resveratrol. J Altern Complement Med 10: 235-239, 2004.
28. Alkhalaf M: Resveratrol-induced apoptosis is associated with activation of $\mathrm{p} 53$ and inhibition of protein translation in T47D human breast cancer cells. Pharmacology 80: 134-143, 2007.

29. Hollstein M, Sidransky D, Vogelstein B, et al: p53 mutations in human cancers. Science 253: 49-53, 1991.

30. Sullivan A and Lu X: ASPP: a new family of oncogenes and tumour suppressor genes. Br J Cancer 96: 196-200, 2007.

31. Harris AL: Mutation p53 the commonest genetic abnormality inhuman cancer. Pathology 162: 5-6, 1990.

32. Weninberg RA: Tumor suppressor genes. Science 254: 1138 1145,1991

33. Liu ZJ, Lu X, Zhand Y, et al: Downregulated mRNA expression of ASPP and the hypermethylation of the 5'-untranslated region in cancer cell lines retaining wild-type p53. FEBS Lett 579: $1587-1590,2005$ 